\title{
A Study on Effect of Music on Working Memory in Healthy Young Adults
}

\author{
Ravi Indrapal Singh ${ }^{1}$, Latika Mohan², Rajesh Kathrotia ${ }^{2, *}$
}

\section{Ravi Indrapal Singh', Latika Mohan², Rajesh Kathrotia ${ }^{2, *}$ \\ ${ }^{1} 3^{\text {rd }}$ year MBBS Student, All India Institute of Medical Sciences (AlIMS), Rishikesh, Uttarakhand, INDIA. ${ }^{2}$ Department of Physiology, All India Institute of Medical Sciences (AlIMS), Rishikesh, Uttarakhand, INDIA.}

\section{Correspondence}

\section{Dr. Rajesh Kathrotia}

Department of Physiology, All India Institute of Medical Sciences (AlIMS), Rishikesh Virbhadra Marg, Rishikesh249203, Uttarakhand, INDIA.

Phone: +91 0135-2462504

Email: rajeshkathrotia@gmail.com

\section{History}

- Submission Date: 23-06-2019

- Review completed: 05-07-2019;

- Accepted Date: 24-07-2019.

DOI : 10.5530/ijcep.2019.6.2.14

\section{Copyright}

(C) 2019 Phcog.Net. This is an openaccess article distributed under the terms of the Creative Commons Attribution 4.0 International license.

\begin{abstract}
Background and Aim: Working memory is divided into two independent subsystems: the phonological loop and the visuospatial sketchpad. In normal day to day life, we may use both these subsystems together as in listening to music while studying. This study aims to investigate whether this interaction is beneficial or not. The present study sought to determine the effect of different types of music on working memory. Methods: 20 Male and 13 young healthy female volunteers were included in the study. They were shown and asked to remember textual and facial information in the presence of different varieties of background music. They were asked to recall as many as they could soon after. Their subjective feelings about the music and their performance while memorizing and recalling for each task was also taken and compared with their original performance. Their performance in presence of different music was also compared. Results: It was found in this study that music had no effect on short term textual memory or face recognition memory, but if the tone played in the background expresses similar emotion as that of the face seen (e.g. joyful music with happy facial expression) it may positively help in memory encoding and recalling as pointed out by our subjective recalling scores. Discussion: While memorizing textual and facial pieces of information, different people respond differently to the music in the background as confirmed by our current study. But while comparing the subjective scores in the presence of different varieties of music, it was found that the emotional tone conveyed by background music can influence subsequent memory for emotional details of an event, indicating the cross-modal nature of memory for emotional aspects of situations.

Key words: Cognition, Emotion and Music, Facial Memory, Short-term Memory, Textual Memory.
\end{abstract}

\section{INTRODUCTION}

While there is literature that affirms that music is a distinct modality from linguistic and other cognitive processing, Research also provides evidence that music influences linguistic and cognitive process. ${ }^{[1,2]}$ In light of this evidence, it is not surprising that educators of all the domains which need memory retention are constantly in search of techniques that can enhance learning, if not accelerate it, at least increase retention of the material taught or read.

According to a current popular model, memory consists of three processes: Echoic memory, Short-term Memory (STM) and Long-term Memory (LTM). Short-term memory is also referred to as Working Memory (WM) and is composed of both processing and storage components. ${ }^{[3]}$ The most prevalent model of STM is divided into two independent subsystems: The "phonological loop" and the "visuospatial sketchpad". ${ }^{[4]}$ Information held in STM is easily lost if not rehearsed and is subject to disruption. More specifically, it has been suggested that the presence of an auditory distracter interferes with the "phonological loop" portion. ${ }^{[5,6]}$ In the presence of verbal cues, the phonological loop is employed in maintaining and rehearsing the information and

similarly visuospatial sketchpad is employed for visual information. In normal day to day life often both verbal and visual information are often presented simultaneously as may happen in listening to music while studying. It therefore becomes relevant to investigate the interaction between visual and auditory information presented at the same time and see if it enhances learning and memory retention. Contrary to the large amount of research done on effect of music on word retention, there are very few studies on the effect of music on facial memory. Available work suggests that listening to emotionally touching music enhances facial memory. ${ }^{[7]}$

Various studies conducted previously on this effect of music or background noise on short term memory, reflect mixed results. It has been shown that unattended speech (speech that is not relatable to the task at hand) as well as vocal music impairs textual memory tasks, ${ }^{[6,8]}$ however, Wallace and his colleagues documented and validated the use of music to enhance memory performance. ${ }^{[9]}$ In other study done by Hodges, music has been shown to strongly affect the learner's attitude, motivation, anxiety level, acculturation, personality and mood. ${ }^{[10]}$ 
Alley and Greene found that vocal music is significantly more detrimental to STM as compared to instrumental music. ${ }^{[1]]}$

A recent study by Proverbio and Benedetto concluded that auditory enhancement of visual memory encoding is driven by emotional content of the auditory material and mediated by superior frontal cortex. ${ }^{[12]}$

In facial memory retention task in the presence of different types of music it is postulated that various other confounding factors may play a role such as type of face, emotional expressions, ethnicity and accessories worn. Specific attention was paid to this aspect in the current study and all such factors were minimised. The hypothesis for facial recognition task in the presence of music remained the same as for word memory, viz, that in the presence of music, due to utilization of different areas of brain the attention of the brain may be divided and the task may be better performed in complete silence.

\section{MATERIALS AND METHODS}

\section{Study Population}

Thirty-three healthy participants (20 males and 13 females) all $2^{\text {nd }}$ year MBBS students, were included in this study. All subjects were Indian and of similar educational status. They were all familiar with Bollywood music. Participants with defects in hearing or vision and who had suffered from previous or current psychiatric or neurological diseases were excluded from the study. Subjects associated with any musical training were also not included in the study.

\section{Study Protocol}

Laptops were installed with 8 Power point files ( 4 for textual memory task and 4 for facial recognition memory task). Music in the background with of each file represented one type of music (4 varieties of music were used in this study). Headphones of JBL T250SI T250 SI Wired were employed for listening to the music while performing facial and textual memory tasks. The same intensity of $25 \mathrm{~dB}$ was used for all types of music. Music which was used during the tasks was based on current general taste of the Indian population. A carefully selected playlist was made of Bollywood music and ambient music. The same was used for all participants.

Participants were explained detailed purpose of the experiment and written informed consent was taken. The subjects were further given detailed verbal instructions on what to do during each session.

\section{Textual Memory Task}

Participants were given thirty seconds to memorize a list of twenty words followed by $15 \mathrm{sec}$ silent latent period, ensued by a maximum of one minute to write down as many words as they could remember (subjects could stop before sixty seconds if they felt they were not able to remember any more words). All the words that were used were randomly chosen without any kind of interconnection with one another.

\section{Facial Memory Task}

Participants were asked to memorize 25 random faces appearing on their laptop screen for 50 seconds. The faces were presented for $800 \mathrm{~ms}$ each with an Interstimulus time (ISI) of $1200 \mathrm{~ms}$. It was followed by a latent period of $15 \mathrm{sec}$ and after which subject were shown 30 faces which will include 10 old faces and 20 new faces for $60 \mathrm{sec}$. Subjects were asked to raise their hand whenever the old faces appear on the screen and their responses were simultaneously recorded. The faces were selected from available, open access, license-free FEI face databases (fei.edu.br/ cet/ facedatabase.html). The faces were equally represented by sex and age ranges. The pictures included different faces up to the base of the neck along with various accessories (e.g., glasses, hats, earrings, etc.) and depicting nearly similar emotional expressions of joy.
The tests were conducted in 8 different sessions ( 4 for textual memory task and 4 for facial recognition task) with a gap of 4 days between two consecutive sessions. In each session all the 33 subjects were made to participate in both visual and textual tasks and the participants were randomly exposed to variety of music.

The 4 varieties of music that we used in our study were

1. Complete silence in an anechoic chamber.

2. Joyful bollywood music.

3. Relaxation music

4. Ambient noise

\section{Recording Subjective Feelings}

A visual analog scale was provided to each participant after completion of the facial and textual memory task, ranging from -6 to +6 and participants were asked to grade their

1. Opinion about the music

2. Performance while memorizing words or faces

3. Performance while recalling words and faces

One music session was graded only once by each participant and that too after their text memorizing session. Only one reading about the subjective opinion about music was taken based on our assumption that opinion about music will not significantly depend upon tasks and will be more or less equal for both the scenarios.

\section{Statistical Analysis}

In the textual memory task in each session, +1 points was awarded for each correct word that they were able to recall and all the points were added to give them a final score.

In facial recognition task for each correct response +1 was given and each incorrect response was given -1 and final score was calculated by adding all the points. Data was expressed mean \pm Standard deviation for normal distributed data and as median for not normal data. Statistical analysis was done using IBM SPSS version 23. Repeated measure ANOVA or Kruskal Walis test was applied for parametric or non-parametric data respectively for comparison. Post hoc Tukey test was used to compare the difference between two groups. Statistical significance was considered at $p<0.05$ and confidence interval of $95 \%$.

\section{RESULTS}

\section{Demographics}

All the participants were volunteers from $2^{\text {nd }}$ year MBBS students of AIIMS Rishikesh on the basis of their interest in the research undertaken. Total thirty-three participants (20 males and 13 females) were included in study. Mean age of participants was $19 \pm 0.84$ years (range 18-22 years).

\section{Text Memorization Results}

The mean of the correct text memorization response was found in the order of relaxation music $(10.3 \pm 2.99)>$ silence $(9.67 \pm 2.17)>$ bollywood music $(9.52 \pm 2.99)>$ ambient noise $(9.15 \pm 2.69)$, hence the frequency of correct response is more in the presence of relaxation music in the background and least in the presence of ambient noise in the background. Using ANOVA to compare the values of the achieved score on the text memorization test, no statistical significance between categories was found.

Median subjective feeling of memorization of the participants as in Figure 1 shows that the order of memorization was of relaxation music (2.7) $>$ silence $(2)>$ bollywood music $(1.4)>$ ambient noise $(-0.3)$. Therefore, the participants claim that they were able to memorize more in the presence of relaxation music (2.7) and least or nearly unable to 


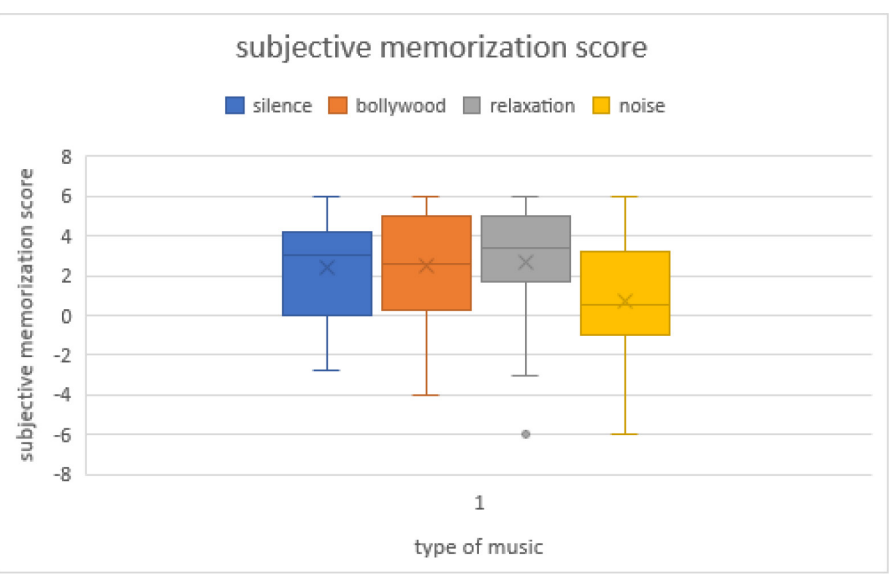

Figure 1: Subjective memorization score for text memorization task.

Table 1: Post hoc Tukey test results for subjective text memorization scores for various combinations of music in the background.

$\begin{array}{cc}\text { Combination } & \text { Result } \\ \text { silence vs. bollywood music } & \text { Non-significant } \\ \text { silence vs. relaxation music } & \text { Non-significant } \\ \text { silence vs. ambient noise } & P<.05 \\ \text { bollywood music vs. relaxation music } & \text { Non-significant } \\ \text { Bollywood music vs. ambient noise } & \text { Non-significant } \\ \text { Relaxation music vs. ambient noise } & P<.01\end{array}$

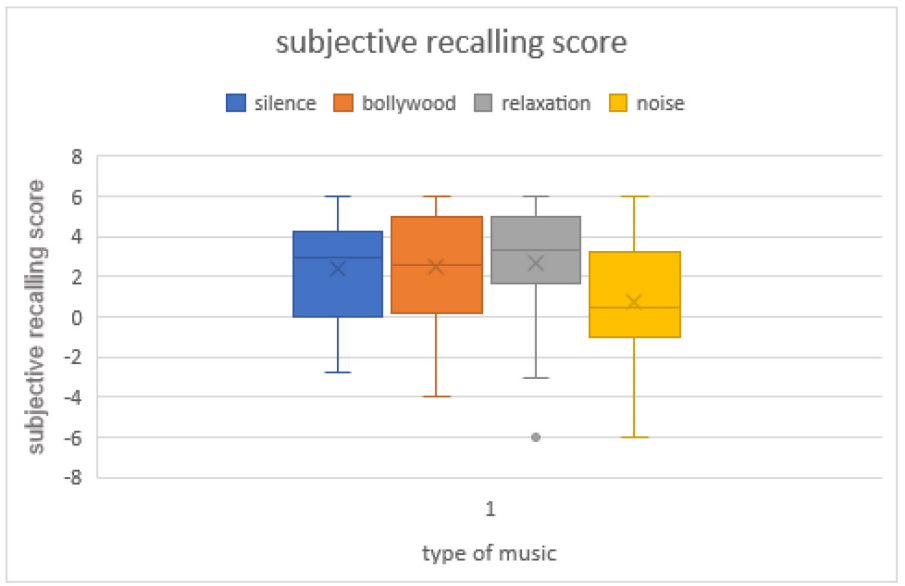

Figure 2: Subjective recalling score for text memorization task.

memorize information in the presence of ambient noise. Applying Kruskal Wallis test to the subjective feeling of memorization in the presence of different music in the background we get the $p=0.0083$ which is statistically significant and applying the post hoc Tukey test for comparing the results of two combinations in various combinations possible we get the results shown in Table 1. Memorization is more in the presence of silence when compared to noise in the background $(p<0.05)$ and the memorization is more in the presence of relaxation music when compared to noise in the background $(p<0.01)$.

Median scores of recalling capacity of the participants in the presence of different music in the background was silence (1.9) = relaxation music (1.9) > bollywood music (1.5) > ambient noise (-0.5) in that order as shown in Figure 2 and applying Kruskal Walis test to the scores we get $p=0.0047$ which is statistically significant and applying Post hoc Tukey to compare two sets at a time we get the results as shown in Table 2.

Post hoc Tukey test indicate recalling in the presence of silence is more when compared to that ambient noise $(p<0.05)$ and also more with relaxation music compared to ambient noise $(p<0.05)$ suggesting better recall in the presence of relaxation music when compared to noise in the background

\section{Face Recognition Results}

The mean of the correct facial recognition response is relaxation music $(8.2 \pm 1.45)>$ bollywood music $(8.03 \pm 1.38)>$ silence $(7.91 \pm 1.58)>$ ambient noise $(7.8 \pm 1.57)$ in that order, so the correct response is more in the presence of relaxation music in the background and least in the presence of ambient noise in the background. Mean of wrong response was in the order ambient noise (2.3) > bollywood music (2.2)> relaxation music (2) $>$ silence (1.7). So, the participants not only gave less correct response but also more wrong responses in the presence of ambient noise in the background. However, on applying ANOVA to correct response $(p=0.73)$ and wrong response $(p=0.65)$ no significant differences was found, going in favor with null hypothesis.

Taking scores of both correct and incorrect responses into consideration, the mean of the total scores of facial recognition response is in the order relaxation music $(6.5 \pm 2.2)>$ silence $(6.2 \pm 2.3)>$ bollywood music (5.9 \pm 2.1$)>$ ambient noise (5.8 \pm 2.8$)$. On Comparing silence and Bollywood music in the background, though the participants gave more correct response in the presence of bollywood music but the high wrong response in its presence masked the overall performance and finally the total response was more in the presence of silence in the background but on applying ANOVA to total response we get $p=0.68$ which is statistically insignificant.

Median of the subjective feeling of memorization is in the order of relaxation music (2.9) > silence (2.7) > bollywood music (2.4) > ambient noise (0.7), so the participants claim that they were able to memorize more in the presence of relaxation music (2.9) and least or nearly unable to memorize information in the presence of ambient noise (0.7) as seen in Figure 3. Applying Kruskal Walis test to the subjective feeling of memorization in the presence of different music in the background we get the $p=0.012$ which is statistically significant and applying the Tukey test for comparing the results of two combinations in various combinations possible as seen in Table 3, we get the following results- memorization is more in the presence of silence when compared to noise in the background $(p<0.05)$ and it is also more in the presence of relaxation music when compared to noise in the background $(p<0.05)$.

From the scores on recalling capacity of the participants in the presence of music we get the order as relaxation $\operatorname{music}(2.7)>$ bollywood music (2.6) > silence $(2.3)>$ ambient noise $(0.8)$ as seen in Figure 4, it shows although the participants claim to memorize better in the presence of

Table 2: Post hoc Tukey test for subjective text recalling scores for various combinations of music in the background.

$\begin{array}{cc}\text { Combination } & \text { Result } \\ \text { silence vs. bollywood music } & \text { Non-significant } \\ \text { silence vs. relaxation music } & \text { Non-significant } \\ \text { silence vs. ambient noise } & P<.05 \\ \text { bollywood music vs. relaxation music } & \text { Non-significant } \\ \text { Bollywood music vs. ambient noise } & \text { Non-significant } \\ \text { Relaxation music vs. ambient noise } & P<.05\end{array}$




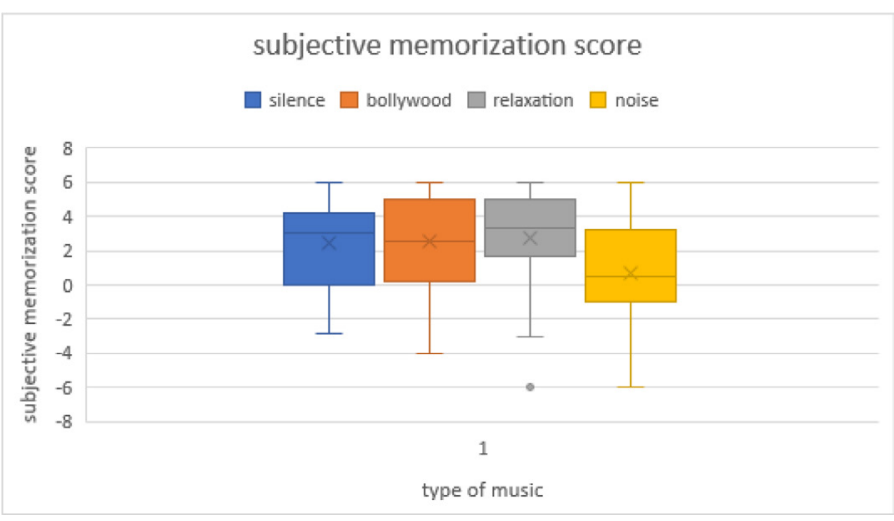

Figure 3: Subjective memorization score for face recognition task.

Table 3: Post hoc-Tukey test for subjective face memorization scores for various combinations of music in the background.

\begin{tabular}{cc} 
Combination & Result \\
silence vs. bollywood music & Non-significant \\
silence vs. relaxation music & Non-significant \\
silence vs. ambient noise & $P<.05$ \\
bollywood music vs. relaxation music & Non-significant \\
Bollywood music vs. ambient noise & Non-significant \\
Relaxation music vs. ambient noise & $P<.05$ \\
\hline
\end{tabular}

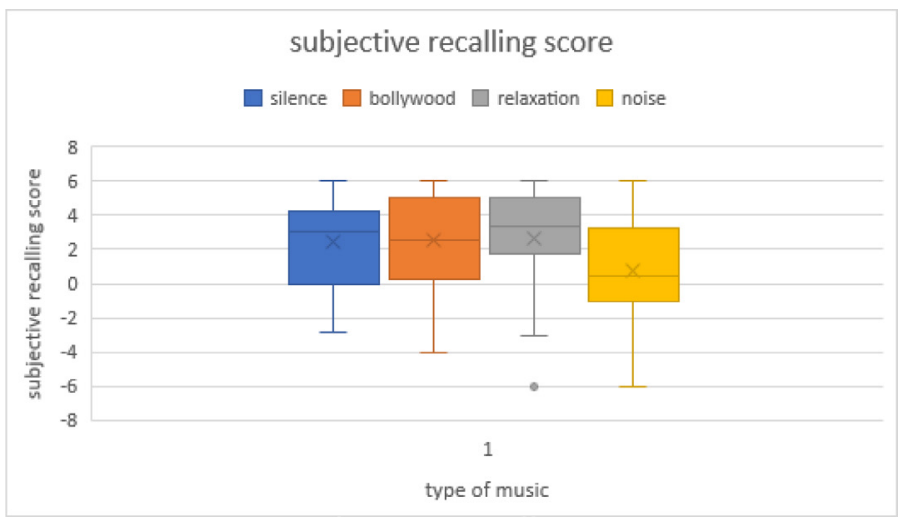

Figure 4: Subjective recalling score for face recognition task.

relaxation music in the background. comparing in between silence and bollywood music in the background the participants feel they can recall better in the presence of bollywood music in contrast to silence in the background. Applying Kruskal Walis test to the subjective feeling of recalling with different background music we get $p=0.022$ which is statistically significant and applying post hoc Tukey to compare two sets at a time we get the results shown in Table 4 .

So, recalling is more in the presence of relaxation when compared to ambient noise $(p<0.05)$ and it is also more in the presence of bollywood music in the background when compared to ambient noise $(p<0.05)$

\section{Subjective Feelings Towards Music}

The median scores of the subjective feeling of the participants towards the music is in the order of relaxation music (5) > bollywood music (2.7) $>$ silence $(0)>$ ambient noise $(-3)$. So, the participants appreciated the
Table 4: Tukey test results for subjective face recalling scores for various combinations of music in the background.

\begin{tabular}{cc} 
Combination & Result \\
silence vs. bollywood music & Non-significant \\
silence vs. relaxation music & Non-significant \\
silence vs. ambient noise & Non-significant \\
bollywood music vs. relaxation music & Non-significant \\
Bollywood music vs. ambient noise & $P<.05$ \\
Relaxation music vs. ambient noise & $P<.05$ \\
\hline
\end{tabular}

Table 5: Post hoc Tukey test results for subjective scores of various combinations of music in the background.

$\begin{array}{cc}\text { Combination } & \text { Result } \\ \text { silence vs. bollywood music } & \text { Non-significant } \\ \text { silence vs. relaxation music } & P<.01 \\ \text { silence vs. ambient noise } & P<.01 \\ \text { bollywood music vs. relaxation music } & P<.05 \\ \text { Bollywood music vs. ambient noise } & P<.01 \\ \text { Relaxation music vs. ambient noise } & P<.01\end{array}$

relaxation music and disliked the ambient noise. On applying Kruskal Walis test to the subjective score given to the varieties of music used in the background we get $p<0.0001$ so the test is highly statistically significant and applying Post hoc Tukey test to compare two varieties of music at a time we see insignificant difference in between silence and Bollywood music and significant differences with other pairs as shown in Table 5

From the overall results we have found that music didn't affect the textual memory or face recognition memory but if the tone played in the background expresses similar emotion as that of the face seen (eg joyful music with happy facial expression) it may positively help in memory encoding and recalling as pointed out by our subjective recalling scores.

\section{DISCUSSION}

The purpose of this study was to determine whether a participant's ability to retain textual and face recognition memory could be altered in response to listening different varieties of music in the background. After analysing the data gathered from the experiment, we concluded our results for facial recognition and textual memory to be insignificant to this study, thus not allowing the hypothesis to be supported. The differences in the scores between the four groups of music supported the idea that music in the background affects the text and face memory retention in general terms but the analysis of data provided no significant differences. This data is in accordance with some of the researches done earlier which tried to determine effect of music tempo on memory retention where no significant relation between short or fast tempo of the music and the memory retention was found. ${ }^{[13]}$ This results shows the variability seen in general population - while one person can retain pieces of information longer in the presence of noise whereas other is unable to do so in the presence of silence as well.

In a study done by Gustavson and his colleagues on effects of music on short-term memory and physiological arousal it was shown that despite a significant increase in sympathetic activity during testing as compared to baseline, subjects did not significantly vary in memory scores ${ }^{[14]}$ which is again in accordance to our findings. So, in conclusion our hypothesis 
fails to explain the memorizing capacity of different people in different background. One reason for this conclusion may also be the difference in the levels of anxiety seen in the population because the mean of the scores of memorizations was higher for relaxation music which may be because the music helped them to calm down their mind and perform better.

In the face recognition task also, the scores showed differences with different background music but failed to show any statistically significant findings. A recent study done by Proverbio and his colleagues to find out the effect of memory on different auditory backgrounds has shown a significant effect of music on memory in which listening to emotionally touching music enhanced the facial memory capacity. ${ }^{[7]}$ But no such effect on memory encoding was seen in our findings although the mean of final scores for relaxation music as well as for silence were highest but the results came out to be statistically insignificant.

Coming to the subjective score it has been shown that students are aware of the impact of music on the both the cognitive and the affective processes which was in accordance with the previous research survey. ${ }^{[15]}$ From the overall varieties that were played in the background a significant score was given to the relaxation music and least to the ambient noise. Showing more preference for the relaxation music may be due to the high stress levels in the college students. Hence as per our hypothesis although the performance of the participants was supposed to decline in the presence of any music including the relaxation music but the improved performance as well greater preference for relaxation music was seen which may be due to their stress levels, hence it may not be only the music which is determining the result but also the stress levels and the anxiety status of the subjects. Further these findings welcomes new research which should be focusing on effective ways of reducing the stress of the participants and thereby improving their performance

Analyzing the subjective scores of memorizations in both the memory tasks significant scores were given to silence and relaxation music in the background when compared to the noise. So, although the participants preferred the relaxation music of all, but the memorization was equal with both silence and relaxation music in the background. As per our hypothesis better performance in the presence of silence was speculated but improved performance in the presence of relaxation music cannot be explained since it was supposed to hinder the memorization capacity of the fellow subjects. The reason which can be given for this finding may be the same difference in the stress level of the subjects, we hypothesize that in some way relaxation music reduced the stress levels of the participants giving them the impression that they are able to memorize better in the presence of relaxation but it has no bearing on the actual test results.

The subjective recalling of the memorized text was highest for silence and relaxation music which is similar to that of the memorization scores for text memorization task. But interestingly for the face recognition tasks the subjective recalling scores were highest for bollywood music and relaxation music in contrast to the higher memorization scores for silence and relaxation music. The mechanism that may underlie this result may be that the music played affected memory for facial expressions presented concurrently either by activating a general emotional schema used to represent the scene, or as a result of a source confusion error during recall. ${ }^{[16]}$ To illustrate, according to a schema explanation the participants thought that they recalled more faces in the background of bollywood was because all the faces that were used in the picture were smiling and joyful and the bollywood music which was used was also joyful so the music paired with the facial expression might cause the information to be encoded more deeply in the memory as being a "happy" memory. A recent study shows auditory enhancement of visual memory encoding is driven by emotional content of the auditory material which supports our findings. ${ }^{[12]}$ But inconsistency of the subjective recalling scores with the total score was because of increased wrong response along with correct responses so as to nullify the effect of one another. It can be deduced that the emotional expressions are encoded in the memory instead of actual faces in the presence of music depicting similar emotions because of which participants got confused between old and new faces. Many studies use vocalizations paired with faces for emotion interpretation. ${ }^{[17,18]}$ So, the current findings extend the dimension of auditory information that can be integrated with facial expressions to influence recall, possibly occurring at the point of encoding or during memory consolidation. In order to verify this finding further research is needed where the researcher would be using varieties of music reflecting different emotions along with the faces depicting various types of emotions and then finding whether similar emotion reflecting in picture as well as in the music is helping to encode the information better or not. Although we have more or less verified this finding for joyful music but whether same effect would be observed for other emotions as well needs further exploration.

The study group was homogenous as they had many things in common such as they were in the same college pursuing the same course and had similar qualification status and in the age range of 18-22 years. However, some factors might distort the result like the test was given at inconsistent times of the day and therefore all participants were in different stages of their sleep-wake cycle and also some participants may be more accustomed to music compared to others. Further studies with more participants and people from different strata of the population with controlled uniform environment may yield novel results.

\section{CONCLUSION}

In summary, these findings suggest different background music do not have any significant effect on the working memory for text recalling and face recognition task while positive subjective recalling was seen for happy faces with joyful Bollywood music suggesting that the emotional tone conveyed by background music can influence subsequent memory for emotional details of an event, indicating the cross-modal nature of memory for emotional aspects of situations.

\section{ACKNOWLEDGEMENT}

We are very thankful to all participants for their valuable time and efforts for this study.

\section{CONFLICT OF INTEREST}

Authors declare that they have no conflict of interest.

\section{ABBREVIATIONS}

ANOVA: Annalysis of Variance; AIIMS: All India Institute of Medical Sciences.

\section{REFERENCES}

1. Moreno S, Marques C, Santos A, Santos M, Castro SL, Besson M. Musical training influences linguistic abilities in 8-year-old children: More evidence for brain plasticity. Cerebral Cortex. 2008;19(3):712-23.

2. DenStock JV, Peretz I, Grezes J, DeGelder B. Instrumental music influences recognition of emotional body language. Brain Topography. 2009;21(3-4):216-20.

3. Atkinson RC, Shiffrin RM. Human memory: A proposed system and its control processes. In Psychology of Learning and Motivation. 1968;2:89-195. Academic Press.

4. Baddeley A. The concept of working memory: A view of its current state and probable future development. Cognition. 1981;10(1-3):17-23.

5. Cocchini G, Beschin N, Jehkonen M. The Fluff Test: A simple task to assess body representation neglect. Neuropsychological Rehabilitation. 2001;11(1):17-31.

6. Salame P, Baddeley A. Disruption of short-term memory by unattended speech: Implications for the structure of working memory. Journal of Verbal Learning 
and Verbal Behavior. 1982;21(2):150-64.

7. Proverbio AM, Nasi VL, Arcari LA, DeBenedetto F, Guardamagna M, Gazzola $M$, et al. The effect of background music on episodic memory and autonomic responses: Listening to emotionally touching music enhances facial memory capacity. Scientific Reports. 2015;5:15219.

8. Salamé P, Baddeley A. Effects of background music on phonological shortterm memory. The Quarterly Journal of Experimental Psychology Section A. 1989;41(1):107-22.

9. Wallace WT. Memory for music: Effect of melody on recall of text. Journal of Experimental Psychology: Learning, Memory and Cognition. 1994;20(6):1471.

10. Hodges DA. Implication of Music and Brain Research: This introductory article offers an overview of neuromusical research and articulate some basic premises derived from this research. Music Educators Journal. 2000;87(2):17-22.

11. Alley TR, Greene ME. The relative and perceived impact of irrelevant speech, vocal music and non-vocal music on working memory. Current Psychology. 2008;27(4):277-89
12. Proverbio AM, DeBenedetto F. Auditory enhancement of visual memory encoding is driven by emotional content of the auditory material and mediated by superior frontal cortex. Biological Psychology. 2017;132:164-75.

13. Jurkovic A, Anderson C, Myklejord D, Levin C, Lotz A. The effect of music tempo on memory retention. J Ass Neuro Wisc Edu Physiol Dep. 2013;602:1-9.

14. Gustavson A, Hanneken K, Moldysz A, Simon B. The Effects of Music on ShortTerm Memory and Physiological Arousal. J Ass Neuro Wisc Edu Physiol Dep. 2014;435:1-8.

15. Jones A. Music and the Cognitive Process-Student Perceptions. Polyglossia. 2010:19:143-50.

16. Johnson MK, Hashtroudi S, Lindsay DS. Source monitoring. Psychological Bulletin. 1993;114(1):3-28.

17. DeGelder B, Vroomen J. The perception of emotions by ear and eye. Cognition and Emotion. 2000;14(3):289-311.

18. Thompson FT, Russo FA, Quinto L. Audio-visual integration of emotional cues in song. Cognition and Emotion. 2008;22(8):1-14.

Cite this article: Singh RI, Mohan L, Kathrotia R. A Study on Effect of Music on Working Memory in Healthy Young Adults. Int J Clin Exp Physiol. 2019;6(2):49-54. 